\title{
Bardet-Biedl syndrome due to a pathogenic mutation on CEP290 and an unreported TTC8 variant
}

\author{
Samantha Karlin¹, Bimota Nambam², Yves Lacassie ${ }^{3 *}$
}

\author{
${ }^{*}$ Correspondence \\ Yves Lacassie \\ Professor Emeritus, Department of \\ Pediatrics, LSU Health Sciences Center \\ and Children's Hospital, 200 Henry Clay \\ Ave., New Orleans, LA 70118, USA \\ Email:ylacas@lsuhsc.edu \\ yves.lacassie@lcmchealth.org \\ 1LSU Health Sciences Center School of \\ Medicine, New Orleans, Louisiana \\ 2Department of Pediatrics, Division of \\ Endocrinology, LSU Health Shreveport, \\ Louisiana \\ ${ }^{3}$ Department of Pediatrics, Division of \\ Genetics, LSU Health Sciences Center \\ and Children's Hospital, New Orleans, \\ Louisiana \\ Received: Mar 21, 2018 \\ Accepted: Apr 6, 2018 \\ Published: Apr 7, 2018

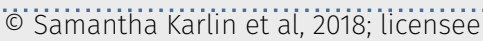 \\ OA Journal of Case Reports. This is an \\ Open Access article distributed under \\ the terms of the Creative Commons \\ Attribution License (http:// \\ creativecommons.org/licenses/by/4.0) \\ which permits unrestricted use, \\ distribution, and reproduction in any \\ medium, provided the original work is \\ properly credited.
}

\begin{abstract}
Bardet-Biedl syndrome (BBS) is a rare autosomal recessive or triallelic ciliopathy disorder characterized by obesity, male hypogonadism, renal impairment, polydactyly, and intellectual disability. We present a case report of an African-American male with excessive weight gain and prediabetes who was referred to Genetics at 21/2 years of age. Physical exam findings included obesity, macrocephaly, bilateral macrotia, brachydactyly, and small genitalia. Gene sequencing identified two mutations highly suggestive of BBS: a heterozygous variant in the CEP290 gene (C.4393 C>T) and a heterozygous variant in the TTC8 gene (c.1021 C>T). Twenty-one genes have been associated with the 21 types of BBS to date, all demonstrating variable expressivity and incomplete penetrance. There is no cure for BBS, and current management focuses on preventing and treating symptoms. CEP290 mutations are associated with BBS type 14, and only $1 \%$ of affected individuals carry a mutation on this gene. TTC8 mutations are associated with BBS type 8 , and its gene frequency is also $1 \%$ in individuals with BBS. The TTC8 variant found in our propositus (p.Arg341Trp) has not been previously reported in the Human Gene Mutation Database. In this variant, tryptophan replaces the normal arginine at position 341 of the TTC 8 protein. To our knowledge, this is the first report linking this specific TTC8 variant with BBS.
\end{abstract}

Keywords: Bardet-Biedl Syndrome, Ciliopathy disorder, Childhood obesity, Genetic obesity, CEP290, TTC8

\section{Background}

Bardet-Biedl syndrome (BBS, OMIM 209900) is a rare autosomal recessive or triallelic multisystem ciliopathy disorder primarily characterized by obesity, male hypogonadism, female genital tract anomalies, renal anomalies, polydactyly, intellectual disability, and retinal dysfunction with rod-cone dystrophy. Secondary manifestations can include diabetes mellitus, hepatic fibrosis, speech delay, developmental delay, behavioral abnormalities, mild hypertonia, poor coordination or imbalance, cardiovascular, hepato-gastric, craniofacial, and orodental anomalies. The phenotype of a child with BBS can vary significantly; consequently, clinical diagnosis requires either four primary traits or three primary and two secondary features [1]. The BBS phenotype slowly evolves during the first ten years of life; most individuals are diagnosed with BBS when they are adolescents or young adults. Those affected have immotile cilia that cause abnormalities in signal transduction pathways. Because of this, characteristics of BBS often overlap with traits from 
other ciliopathies, such as Meckel and Joubert syndromes [2].

\section{Case Report}

We report an African-American male with excessive weight gain (weight $Z$ score +7.6) and prediabetes (HbA1c 6\%) who was referred to Genetics at $2 \frac{1}{2} 2$ years of age. According to his mother, the propositus began to gain weight around 9 months of age shortly after switching from formula to whole milk. The weight gain was gradual at first, but then increased dramatically from $10 \mathrm{~kg}$ at age 1 to $28 \mathrm{~kg}$ at age 2. At this time, his pediatrician suggested that he switch to $1 \%$ milk and begin a low-carbohydrate diet. The propositus was followed-up by endocrinology at age $21 / 2$ at which time he weighed $35.8 \mathrm{~kg}$ and his height was $95.3 \mathrm{~cm}$ (BMI 39). His mother reported that he lost some weight initially after implementation of the diet, but then started to gain weight rapidly despite being active and limiting carbohydrates and/or sugary drinks. The child did not have polydipsia, polyuria (excessively wet or heavy diapers), or hyperphagia. He had severe acanthosis nigricans around his neck. Suspecting a genetic syndrome, the endocrinologist obtained gene sequencing for a diabetes/obesity panel and referred to Genetics.

\section{Family History}

Review of history revealed that the propositus was born to a healthy, 39 year-old African-American father and to a 34 year-old G1P1 African-American mother with sickle cell trait, diabetes, and a significantly high BMI. The mother's family had multiple members with diabetes and obesity on both the maternal and paternal sides. The mother also experienced ocular problems with thinning of her left optic nerve. The paternal family history revealed diabetes, hypertension, and an obese 12 year-old nephew with some intellectual disability. The father also had a 10 year-old daughter from a previous relationship whose weight was within normal limits. There was no consanguinity.

\section{Birth and Medical History}

The pregnancy was unplanned and first detected at 16 weeks gestational age. The mother began receiving prenatal care at 20 weeks when she noticed fetal activity. During the pregnancy, the mother had hypertension and type 2 diabetes mellitus (HbA1c 7.9\%). During pregnancy, the mother received subcutaneous insulin, hydrochlorothiazide $25 \mathrm{mg}$ daily, and metformin 1000 mg twice daily; she gained less than $4.5 \mathrm{~kg}$, did not have any infections, and did not smoke, consume alcohol, or use illict drugs. Due to non-reassuring fetal heart tones and breech positioning, the propositus was born at 34 weeks gestational age via emergency Cesarean section. His birth weight was $3.05 \mathrm{~kg}$ (95th centile) and birth length was $50 \mathrm{~cm}$ (98th centile). At birth, he was acrocyanotic, had poor tone, low activity, and minimal crying with stimulation. He spent 5 days in the NICU due to prematurity, hypoglycemia, respiratory distress, and the presence of a heart murmur; however, he required ventilator support, received antibiotics for possible sepsis, and phototherapy for hyperbilirubinemia only on the first day. At $21 / 2$ years of age, the propositus was relatively healthy despite his prediabetes and excessive weight gain. Developmentally, he held his head up between 3 and 4 months, sat up at 5 months, and walked at 9 months; however, he had a speech delay.

\section{Physical Exam}

On physical examination, height was $100 \mathrm{~cm}$ (97th centile); weight was $37.5 \mathrm{~kg}$ (well above 97th centile, corresponding to the 50th centile for 11 years); and head circumference measured $53.3 \mathrm{~cm}$ (above 97th centile for age and height). Besides the obesity, speech delay, and macrocephaly, we noticed bilateral macrotia (75th to 97 th centile for head circumference), small genitalia, and four café-au-lait spots. His palms were large even for height and although his fingers were between the 75th and 97th centile, the finger-to-palm proportion was between the $3 \mathrm{rd}$ and 25 th centile with tendency to brachydactyly. Axial triradius was proximal on the left palm and intermediate on the right. He only spoke in monosyllables and also exhibited some degree of age inappropriate disinhibitory behavior.

\section{Suggested Diagnosis}

The findings on physical exam were suggestive of Bardet-Biedl syndrome (Fig. 1). Given the age of the propositus, he did not yet have four primary BBS features or three primary and two secondary features required to make a definitive clinical diagnosis. However, he exhibited one primary feature of BBS (obesity), as well as small genitalia, commonly seen in infant males with BBS and suggestive of future male hypogonadism; in addition, he had secondary 
features including speech delay and pre-diabetes, suggestive of future diabetes mellitus. Often, the clinical scenario is incomplete given many defects that contribute to primary traits of BBS (renal, ocular, gonadal) are discovered later in life. This clinical suggestion of BBS, however, aligned with the results of the diabetes/obesity panel obtained at Fulgent Diagnostics Lab, which sequenced 56 genes including large deletions and duplications. Results indicated two mutations highly suggestive of BBS: a heterozygous variant in the CEP290 gene (c.4393 C>T) and a previously unreported heterozygous variant in the TTC8 gene (c.1021 C>T). The genetic study of the parents and segregation studies could not be performed.

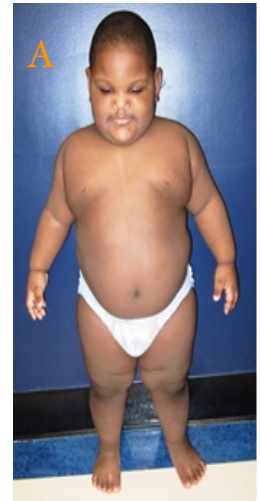

A: Whole body

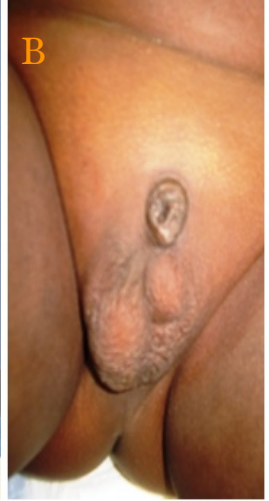

B: Small genitalia C: Relative brachydactyly Fig 1. Photograph of Propositus at age $2 \frac{1}{2} 2$ years.

\section{Discussion}

To date, 21 causative genes have been associated with BBS (types 1-21), all showing variable expressivity and incomplete penetrance [3]. Most individuals with BBS have mutations associated with BBS types 1 and 10, while the other nineteen types are seen less frequently, some reported in single families [4]. The mode of inheritance is usually autosomal recessive, although the diversity in presentation has been attributed to rarer cases of triallelic inheritance, in which the affected individual displays three mutations in two different genes [1].

\section{CEP290 Gene in BBS}

The CEP290 gene codes for a centrosomal protein [4]. Biallelic mutations in the CEP290 gene have been associated with BBS type 14, Joubert syndrome, Leber congenital amaurosis, Meckel syndrome, and SeniorLoken syndrome []‥ Mutations in this gene affects organization of ciliary entry and transition within the
BBSome [4]. Only $1 \%$ of individuals with BBS carry a mutation on this gene [2]. The specific variant found in our propositus (p.Arg1465*) is a known pathogenic nonsense mutation that has only been observed at a frequency of less than $0.01 \%$ in individuals without severe childhood onset disease (Broad ExAc dataset).

\section{TTC8 Gene in BBS}

TTC8 mutations are mainly seen in affected individuals of Middle Eastern or North African descent and have been associated with BBS type 8 . Although infrequently reported, TTC8 is also a causative gene for nonsyndromic autosomal recessive retinitis pigmentosa [6]. Similar to CEP290, its frequency is also $1 \%$ in individuals with BBS [2]. Interestingly, the variant in our propositus (p.Arg341Trp) has not been previously reported in the Human Gene Mutation Database. In this variant, tryptophan replaces the normal arginine at position 341 of the TTC8 protein. Conservation of the wild-type arginine is seen in all vertebrates on amino acid conservation analysis, implying that any change in this sequence would cause a deleterious effect (Human, December 2013, GRCh38/hg38 Assembly).

\section{Hybrid of BBS Types}

BBS type 8 is characterized by obesity, retinitis pigmentosa, polydactyly of the hands and feet, brachycephaly, male hypogonadism, hypospadias, and developmental delay. Situs inversus also has occasionally been documented [7]. Similarly, BBS type 14 is characterized by obesity, retinitis pigmentosa, developmental delay, and renal anomalies; however, there is absence of polydactyly, brachycephaly, and hypogonadism [8]. Our propositus exhibits features of both BBS type 8 and 14 , as well as some individual features (Table 1). As more than $90 \%$ of patients with BBS have rod-cone dystrophy which becomes clinically apparent by 7-8 years of age and worsens as one reaches adolescence, electroretinogram (ERG) is recommended for all patients with BBS [9]. However, as significant rod-cone dystrophy is not apparent under 5 years of age, and there is poor cooperation with testing in younger children, ERG is not recommended before 4 years of age unless there is a strong indication for earlier testing. Our patient has not shown signs of visual impairment even at night, but did not cooperate during his initial ophthalmologic evaluation; an elective ERG is 
planned in the future. Echocardiogram and ultrasound of kidneys have been normal, and he will continue to follow-up with pediatric cardiology and nephrology. He has bilateral pes planus and is currently undergoing evaluation by pediatric orthopedics.

Table 1. Comparison of Clinical Findings of BBS types 8 and 14 and the Propositus

\begin{tabular}{|l|c|c|c|}
\hline & $\begin{array}{c}\text { BBS } \\
\text { type 8 }\end{array}$ & $\begin{array}{c}\text { BBS } \\
\text { type 14 }\end{array}$ & Propositus \\
\hline Inheritance & AR & AR & $\begin{array}{c}\text { New } \\
\text { mutation }\end{array}$ \\
\hline Mutation & TTC8 & CEP290 & $\begin{array}{c}\text { CEP290 with } \\
\text { TTC8 variant }\end{array}$ \\
\hline Common Manifestations: & + & + & + \\
\hline Obesity & + & + & TBD \\
\hline Retinitis Pigmentosa & - & + & - \\
\hline Renal Disease & + & - & - \\
\hline Polydactyly Hands/Feet & - & - & + \\
\hline Brachydactyly of Fingers & - & - & - \\
\hline Brachydactyly of Toes & + & + & + \\
\hline Developmental Delay & + & + & TBD \\
\hline Intellectual Disability & + & - & TBD \\
\hline Cognitive Impairment & + & - & - \\
\hline Brachycephaly & - & - & + \\
\hline Macrocephaly & - & - & + \\
\hline Macrotia (Bilateral) & + & - & TBD \\
\hline Hypogonadism & + & - & - \\
\hline Hypospadias & & & - \\
\hline Occasional findings: & + & - & - \\
\hline Renal Dysplasia & + & - & - \\
\hline Situs Inversus & & \\
\hline TBD to be determined & & + & \\
\hline
\end{tabular}

$\mathrm{TBD}=$ to be determined

\section{Conclusion}

There is no cure for BBS. Current treatment focuses on reducing symptoms and monitoring for complications. Individuals with BBS require on-going ophthalmologic evaluations and monitoring of blood pressure, renal function, and endocrine and lipid profiles. Management of intellectual disability includes early intervention, special education, and speech therapy as needed. Visual aids and mobility training may be helpful in mitigating progressive vision loss. Individuals with BBS are also at increased risk for diabetes and associated comorbidities. Education, strict nutrition counseling, behavioral therapies, and exercise regimens may help reduce the risks of obesity, diabetes, and heart disease [10,11]. Renal impairment, such as proteinuria and glomerulonephritis, can occur, so patients may benefit from continuous nephrology follow-up. Although current management focuses on preventing and treating symptoms, continued research and advancements in genome sequencing have led to better diagnostic accuracy and novel, potential future therapies such as gene therapy, exon skipping therapy, nonsense suppression therapy, and gene editing [12].

In summary, we report an infant with rapid and excessive weight gain in which gene sequencing revealed a pathogenic mutation of CEP290 and an unreported TTC8 variant highly suggestive of BBS. To our knowledge, this would be the first report linking this specific TTC8 variant with BBS. We hope that this publication will increase awareness of the syndrome and of this previously unknown TTC8 variant.

\section{Acknowledgement}

We thank the family for their cooperation with this publication and Kelly Allerton for the editing.

None of the authors declare any conflicts of interest. There are no funding sources.

\section{References}

1. Forsythe E, Beales PL (2013) Bardet-Biedl syndrome. Eur J Hum Genet 21: 8-13.

2. Suspitsin EN, Imyanitov EN (2016) Bardet-Biedl syndrome. Mol Syndromol 7: 62-71.

3. https://www.omim.org/entry/209900\#46

4. Zaghloul NA, Katsanis N (2009) Mechanistic insights into Bardet-Biedl syndrome, a model ciliopathy. J Clin Invest 119 428-437.

5. https://www.omim.org/entry/610142.

6. Goyal S, Jäger M, Robinson PN, et al. (2015) Confirmation of TTC8 as a disease gene for nonsyndromic autosomal recessive retinitis pigmentosa (RP51). Clin Genet 88: 10.1111/cge.12644.

7. https://www.omim.org/clinicalSynopsis/615985?highlight=bbs8

8. https: / / w w w. om i m.org/entry/615991? search=BBS14\&highlight=bbs14

9. Weihbrecht K, Goar WA, Pak T, et al. (2017) Keeping an eye on Bardet-Biedl Syndrome: a compreshensive review of the role of Bardet-Biedl Syndrome Genes in the eye. Med Res Arch 5: 1-22.

10. Priya S, Nampoothiri S, Sen P, et al. (2016) Bardet-Biedl syndrome: Genetics, molecular pathophysiology, and disease management. Indian J Ophthalmol 64: 620-627.

11. Singh MK, Shrinkhal, Pradhan S, et al. (2017) Bardet Biedl syndrome - A report of two cases with otolaryngologic symptoms. J Clin Diagn Res 11: N01-ND02.

12. Forsythe E, Kenny J, Bacchelli C, et al. (2018) Managing BardetBiedl syndrome - now and in the future. Front Pediatr 6: 23. 